\title{
UNIVERSAL BOUNDS FOR POSITIVE MATRIX SEMIGROUPS
}

\author{
L. LIVSHITS, G. MACDONALD, L. MARCOUX, AND H. RADJAVI
}

\begin{abstract}
We show that any compact semigroup of $n \times n$ positive matrices is similar (via a positive diagonal similarity) to a semigroup bounded by $\sqrt{n}$. We give examples to show this bound is best possible. We also consider the effect of additional conditions on the semigroup and obtain improved bounds in some cases.
\end{abstract}

\section{INTRODUCTION}

It is an old and well-known result (originally shown by Auerbach [1]) that if $\mathcal{G}$ is a compact group of $n \times n$ (real or complex) matrices, then $\mathcal{G}$ is (simultaneously) similar to a group of unitary matrices. In particular, as unitaries act as invertible isometries with respect to the usual operator norm on $\mathbb{M}_{n}(\mathbb{R})\left(\operatorname{resp} . \mathbb{M}_{n}(\mathbb{C})\right)$, we see that given such a group $\mathcal{G}$, it is similar to a group whose elements are uniformly bounded in (operator) norm by 1.

In [6], the first, second and fourth authors obtained a corresponding result for compact semigroups of $n \times n$ matrices by showing that if $\mathcal{S}$ is a compact semigroup of (real or complex) $n \times n$ matrices, then there exists an invertible $n \times n$ matrix $R$ such that with

$$
\mathcal{T}=R^{-1} \mathcal{S} R:=\left\{R^{-1} S R: S \in \mathcal{S}\right\}
$$

we find that

$$
\|\mathcal{T}\|=\max \{\|T\|: T \in \mathcal{T}\} \leq \sqrt{n} .
$$

Furthermore, this bound is optimal in the sense that there exist compact semigroups $\mathcal{S}$ in $\mathbb{M}_{n}(\mathbb{R})\left(\right.$ or $\left.\mathbb{M}_{n}(\mathbb{C})\right)$ for which

$$
\inf \left\{\left\|R^{-1} \mathcal{S} R\right\|: R \text { invertible }\right\}=\sqrt{n} .
$$

It is also shown in [6], that under additional assumptions on the semigroup $\mathcal{S}$ the bound can be improved.

In this paper we consider analogous problems for semigroups of positive matrices (matrices whose entries are non-negative).

The similarities which preserve positivity (i.e. the invertible matrices $X$ for which $X^{-1} A X$ is positive whenever $A$ is positive) are those of the form $X=D P$ where $D$ is a diagonal matrix with positive diagonal entries and $P$ is a permutation matrix. Since permutation matrices are norm-preserving, the universal bound problems analogous to those answered in [6] (there, for the case of general semigroups

Date: February 15, 2016.

1991 Mathematics Subject Classification. Primary 15A30 Secondary 15A21.

Key words and phrases. semigroup, positive, matrix, diagonal similarity, norm bounds.

1. First author's research was supported by Colby College Natural Sciences Division Grant.

2. The other authors' research was supported by NSERC (Canada) Discovery Grants . 
- i.e. semigroups not necessarily consisting of positive matrices), one should consider similarity under a restricted set of invertible matrices: the positive diagonal matrices.

There is a well-known result in the group case here as well. If $\mathcal{G}$ is a compact group of positive $n \times n$ matrices, then $\mathcal{G}$ is (simultaneously) similar (via a positive diagonal similarity) to a group of permutation matrices. (This is an easy consequence of the Perron-Frobenius Theorem, and a self-contained proof can be found as Lemma 5.1.11 of [9].) As permutation matrices are invertible isometries as well, we see that any such group is similar (via a positive diagonal similarity) to a group whose elements are uniformly bounded in (operator) norm by 1 .

What about the corresponding questions for semigroups of positive matrices?

(1) Given a compact semigroup $\mathcal{S}$ of positive $n \times n$ matrices, for what values of $K_{\mathcal{S}}>0$ do there exist a positive invertible diagonal matrix $D$ such that

$$
\sup \left\{\left\|D^{-1} S D\right\|: S \in \mathcal{S}\right\} \leq K_{\mathcal{S}} ?
$$

(2) Do there exist universal constants $K_{n}$ (independent of the semigroup), for each $n=1,2, \ldots$, such that for each compact semigroup $\mathcal{S}$ of positive $n \times n$ matrices, we have a positive invertible diagonal matrix $D$ such that

$$
\sup \left\{\left\|D^{-1} S D\right\|: S \in \mathcal{S}\right\} \leq K_{n} \text { ? }
$$

Also, if such universal constants do exist, what is the best value of $K_{n}$ ?

Perhaps surprisingly, in many cases, the answer for the general semigroups using general similarities - and for the positive semigroups - using positive diagonal similarities - are the same, despite the difference in structure of the semigroups and the difference in the methods used to obtain the results.

Before proceeding, we provide a list of basic definitions and notations used.

\section{Definition 1.1.}

- A matrix $A=\left[a_{i, j}\right]_{i, j=1}^{n}$ is said to be positive if each entry is non-negative $\left(a_{i, j} \geq 0\right.$ for all $\left.i, j=1,2,3 \ldots n\right)$. A set of matrices is positive if each matrix in the set is positive. The set of all positive $n \times n$ matrices will be denoted by $M_{n}\left(\mathbb{R}^{+}\right)$.

- A semigroup of $n \times n$ matrices is a set $\mathcal{S}$ in $M_{n}(\mathbb{R})$ which is closed under matrix multiplication.

- The standard basis of $\mathbb{R}^{n}$ is the set of vectors $\left\{e_{i}\right\}_{i=1}^{n}$, where $e_{i}$ is the vector in $\mathbb{R}^{n}$ with a one in the $i$-th entry and zeros elsewhere.

- A standard subspace is a subspace of $\mathbb{R}^{n}$ spanned by some subset of the standard basis.

- A semigroup $\mathcal{S}$ in $M_{n}(\mathbb{R})$ is indecomposable if it has no invariant standard subspaces other than $\{0\}$ and $\mathbb{R}^{n}$. If a semigroup is not indecomposable, then it is called decomposable.

- A semigroup $\mathcal{S}$ in $M_{n}(\mathbb{R})$ is monomial (resp. submonomial if for each $S \in \mathcal{S}$ exactly (resp. at most) one entry of any row or column of $S$ is non-zero.

- The $\left(\ell_{2}\right)$ norm of a vector $x$ in $\mathbb{R}^{n}$ is denoted $\|x\|$ and is the square root of the sum of squares of its entries. The (operator) norm of a matrix $A$ in $M_{n}(\mathbb{R})$ is

$$
\|A\|=\max \{\|A x\|:\|x\| \leq 1\}
$$


which is the norm of a largest vector in the image (under A) of the unit ball in $\mathbb{R}^{n}$.

- The spectral radius $\rho(A)$ of a matrix $A$ in $M_{n}(\mathbb{R})$ is the modulus of the largest eigenvalue and is also given by

$$
\rho(A)=\lim _{k \rightarrow \infty}\left\|A^{k}\right\|^{1 / k} .
$$

In Section 2, we give an affirmative answer to question (2) above, showing that every compact semigroup of positive matrices is similar (via a positive diagonal similarity) to a semigroup which is bounded in norm by $\sqrt{n}$.

In Section 3, we consider compact semigroup of positive matrices with additional conditions (such as commutivity, self-adjointness, rank conditions, etc.) and in some cases we obtain strict improvements to the bound $\sqrt{n}$.

\section{Universal Bound Theorem}

One of our main results is the following.

Theorem 2.1. If $\mathcal{S}$ is a compact semigroup in $M_{n}\left(\mathbb{R}^{+}\right)$, then there is an $n \times n$ positive diagonal matrix $D$ such that $D^{-1} \mathcal{S} D$ is bounded by $\sqrt{n}$.

We will need a few basic facts about positive vectors:

(1) the usual $\left(\ell_{2}\right)$ norm on $\mathbb{R}^{n}$ is monotonic, in the sense that: for vectors $x$ and $y$ in $\mathbb{R}^{n}$, if $0 \leq x \leq y$ (entrywise) then $\|x\| \leq\|y\|$;

(2) for $x$ and $y$ vectors in $\mathbb{R}^{n}$, with $0 \leq x \leq y$ and $S$ a positive $n \times n$ matrix, $S x \leq S y$

(3) if, for each $x$ in $\mathbb{R}^{n}$ we let $|x|$ denote the vector in $\mathbb{R}^{n+}$ whose entries are the absolute values of the corresponding entries of $x$, then for any positive matrix $S$,

$$
\|S x\| \leq\|S|x|\|
$$

(so positive matrices achieve their norms at positive vectors) and

$$
S|x+y| \leq S|x|+S|y| \text { for all } x, y \in \mathbb{R}^{n} \text { and } S \in \mathcal{S} .
$$

Another key component of our proof is the Fritz John Theorem [4] on symmetric convex bodies. A symmetric convex body $K$ is a bounded convex set in $\mathbb{R}^{n}$ with non-empty interior and with the property that if $x \in K$ then $-x \in K$. The Fritz John Theorem relates such sets to ellipsoids.

Theorem 2.2 (Fritz John [4] ). Let $K \subset \mathbb{R}^{n}$ be a symmetric convex body. Then there is a unique ellipsoid $E \subseteq K$ of maximum volume and for this ellipsoid, $K \subseteq$ $\sqrt{n} E$.

Proof of Theorem 2.1. With no loss of generality we assume that the identity matrix $I$ is in $\mathcal{S}$. Then we define a new norm $\|\cdot\|_{\mathcal{S}}$ on $\mathbb{R}^{n}$ as follows: for $x \in \mathbb{R}^{n}$ let

$$
\|x\|_{\mathcal{S}}=\sup _{S \in \mathcal{S}}\|S|x|\| .
$$

Using the basic facts mentioned above (especially (3)), it is easy to verify that this is a norm on $\mathbb{R}^{n}$.

All norms on $\mathbb{R}^{n}$ are equivalent, so the unit ball of this new norm,

$$
\mathcal{B}=\left\{x \in \mathbb{R}^{n}:\|x\|_{\mathcal{S}} \leq 1\right\},
$$


is a compact, convex set with non-empty interior. Clearly $y \in \mathcal{B}$ implies $-y \in \mathcal{B}$ so $\mathcal{B}$ is a symmetric convex body. Since $\mathcal{S}$ is a semigroup, $S(\mathcal{B}) \subseteq \mathcal{B}$ for all $S \in \mathcal{S}$.

It is immediate from the definition that $\mathcal{B}$ is also invariant under an application of any diagonal matrix with diagonal entries 1 or -1 . Hence the ellipsoid $\mathcal{E}$ in $\mathcal{B}$ (from the Fritz John Theorem) is also invariant under an application of any diagonal matrix with diagonal entries 1 or -1 (i.e. reflections in the standard axes), and hence is a standard ellipsoid (all its axes are in the direction of standard vectors). Of course, also by the Fritz John Theorem:

$$
\mathcal{E} \subset \mathcal{B} \subset \sqrt{n} \mathcal{E} .
$$

Any ellipsoid in $\mathbb{R}^{n}$ which is centered at the origin (like the Fritz John ellipsoid) is an image of the unit ball (in the usual $\ell_{2}$ ) norm under an invertible matrix $X$. With no loss of generality we may assume that $X$ is positive definite, since (by polar decomposition), $X=D U$ where $D$ is positive definite and $U$ is unitary, but the unitary part leaves the unit ball invariant). Since $\mathcal{E}$ is a standard ellipsoid, the positive definite invertible $D$ can be taken to be a diagonal matrix (whose diagonal entries are the stretching factors required in each standard direction to deform the unit ball into $\mathcal{E})$.

So if $B_{1}=\left\{x \in \mathbb{R}^{n}:\|x\| \leq 1\right\}$, then we have that $D\left(B_{1}\right)=\mathcal{E}$.

Now, apply the similarity corresponding to this diagonal $D$ to our semigroup. For any $S$ in $\mathcal{S}$,

$$
\begin{aligned}
D^{-1} S D B_{1} & =D^{-1} S \mathcal{E} \subseteq D^{-1} S \mathcal{B} \\
& \subseteq D^{-1} \mathcal{B} \subseteq D^{-1} \sqrt{n} \mathcal{E}=\sqrt{n} B_{1} .
\end{aligned}
$$

Hence $\left\|D^{-1} S D\right\| \leq \sqrt{n}$ for all $S \in \mathcal{S}$ and the Theorem is proven.

Example 2.1. If we let $[0,1]^{n}=\left\{y \in \mathbb{R}^{n}: 0 \leq y_{i} \leq 1\right.$, for $\left.i=1,2, \ldots, n\right\}$ then it can be shown that the positive semigroup

$$
\mathcal{S}_{[0,1]^{n}}=\left\{S \in M_{n}\left(\mathbb{R}^{+}\right): S[0,1]^{n} \subseteq[0,1]^{n}\right\}
$$

is a compact semigroup of norm $\sqrt{n}$ whose norm cannot be lowered by a positive diagonal similarity.

In fact, if we let $\mathbb{1}_{n}$ denote the vector in $\mathbb{R}^{n}$ with all entries equal to 1 , then

$$
\mathcal{F}_{n}=\left\{e_{i} \mathbb{1}_{n}^{*}: i=1,2, \ldots, n\right\}
$$

is a finite subsemigroup of $\mathcal{S}_{[0,1]^{n}}$ which clearly has norm bound $\sqrt{n}$. If we applied a positive diagonal similarity $D=\operatorname{diag}\left(\alpha_{1}, \alpha_{2}, \ldots, \alpha_{n}\right)$ to this semigroup we would obtain the semigroup

$$
\left\{\alpha_{i}{ }^{-1} e_{i} \alpha^{*}: i=1,2, \ldots, n\right\}
$$

where $\alpha^{*}=\left(\alpha_{1}, \alpha_{2}, \ldots, \alpha_{n}\right)$. Considering norms of elements of this new semigroup we see that

$$
\left\|D^{-1} \mathcal{F}_{n} D\right\|^{2}=\max _{i}\left\|\alpha_{i}{ }^{-1} e_{i} \alpha^{*}\right\|^{2}=\max _{i} \frac{1}{\alpha_{i}{ }^{2}} \sum_{j=1}^{n} \alpha_{j}^{2}
$$

If $D$ reduced the norm below $\sqrt{n}$ we would have that

$$
\sum_{j=1}^{n} \alpha_{j}^{2}<n \alpha_{i}^{2}
$$

for all $i=1,2, \ldots, n$. Summing both sides over such $i$ shows this is impossible. 


\section{BOUNDS UNDER ADDITIONAL CONDITIONS ON THE SEMIGROUP}

As in the general (non-positive) case, we have a dichotomy based on the minimal rank of the semigroup. Semigroups containing rank-one matrices are not (in general) similar to semigroups whose norm is less than $\sqrt{n}$, while groups of invertibles are always similar to groups whose norm is equal to 1 . There is evidence to support the conjecture that for semigroups of constant rank $r$, the higher $r$, the lower the norm bound that can be achieved. The following example hints at the relation between $r$ and the minimal norm bound that can be achieved.

Example 3.1. Let $r$ be an natural number less than or equal to $n$ and let $\mathcal{T}_{r}$ denote the semigroup in $M_{n}\left(\mathbb{R}^{+}\right)$consisting all matrices $T$ with the following properties.

With respect to the decomposition $\mathbb{R}^{n}=\mathbb{R}^{n-r+1} \oplus \mathbb{R} \oplus \mathbb{R} \oplus \cdots \oplus \oplus \mathbb{R}$ ( $r$ direct summands) :

(1) $T$ is sub-monomial (that is, the matrix of $T$ with respect to the above decomposition has at most one non-zero entry in any row or column),

(2) $T_{11}$ (the $(n-r+1) \times(n-r+1)$ block) is from $\mathcal{F}_{n-r+1}=\left\{e_{i} \mathbb{1}^{*}: i=\right.$ $1,2, \ldots, n\} \cup\{0\}$

(3) $T_{1, j}$ for $j=2,3, \ldots, r$, is from $\left\{e_{j}: j=1,2, \ldots, n-r+1\right\} \cup\{0\}$,

(4) $T_{i, 1}$ for $i=2,3, \ldots, r$, is either $\mathbb{1},\{0\}$,

(5) $T_{i, j}$ for $i, j=2,3, \ldots, r$ is either 0 or 1 ,

(where the $\{0\}$ indicates a zero matrix of the appropriate size.)

Then it is easily seen that this is a finite (hence compact) positive semigroup, and since it includes the block diagonal semigroup

$$
\mathcal{F}_{n-r+1} \oplus\left\{I_{r-1}\right\}
$$

it follows from the argument in Example 2.1 that its norm can not be reduced below $\sqrt{n-r+1}$ by a positive diagonal similarity.

Conjecture 3.1. If $\mathcal{S}$ is a compact positive semigroup in $M_{n}\left(\mathbb{R}^{+}\right)$with $\operatorname{rank}(S)=r$ for all $S$ in $\mathcal{S}$, then there exists a positive diagonal invertible matrix $D$ such that $D^{-1} \mathcal{S} D$ is bounded by $\sqrt{n-r+1}$.

One other case of interest is when our semigroup is singly generated and indecomposable. Then, with a little work after using the Perron-Frobenius Theorem (see [8], [3], or see [9] for a more modern treatment), it can be shown that we can achieve a bound of norm 1 after a diagonal similarity. It turns out that the hypothesis of being singly generated can be significantly weakened and we can still achieve the same bound.

We need a two preliminary lemmas before stating our general theorem in this case. These lemmas are well known among mathematicians who work with positive matrices, but we include their proofs for completeness.

Lemma 3.2. If $\mathcal{S}$ in $M_{n}\left(\mathbb{R}^{+}\right)$is an indecomposable semigroup and $\rho(S)=1$ for all $S \in \mathcal{S}$, then $\mathcal{S}$ is bounded.

Proof. If $\mathcal{S}$ is not bounded then there exist $\left\{S_{n}\right\}_{n=1}^{\infty}$ in $\mathcal{S}$ and some $(i, j)$ such that $\left(S_{n}\right)_{i, j} \rightarrow \infty$. But $\mathcal{S}$ is indecomposable so there exists $T$ in $\mathcal{S}$ such that $T_{j, i}>0$. Then consider

$$
\left(S_{n} T\right)_{i, i} \geq\left(S_{n}\right)_{i, j} T_{j, i}
$$


For some $n$ we must have that

$$
\left(S_{n} T\right)_{i, i}=\alpha>1
$$

and hence, taking powers, $\left(S_{n} T\right)^{m}$ has a diagonal entry larger than $\alpha^{m}$. But then

$$
\rho\left(S_{n} T\right)=\lim _{m \rightarrow \infty}\left\|\left(S_{n} T\right)^{m}\right\|^{1 / m} \geq \lim _{m \rightarrow \infty}\left(\left(S_{n} T\right)_{i, i}^{m}\right)^{1 / m} \geq \alpha>1,
$$

a contradiction.

Lemma 3.3. If $A$ in $M_{n}\left(\mathbb{R}^{+}\right)$is such that there exists $x \in \mathbb{R}^{n}$ with all entries of $x$ strictly positive and $A x=x$ then $\rho(A)=1$.

Proof. Take a maximal chain of standard invariant subspaces $\left\{N_{i}\right\}$ for $A$ and then apply Perron-Frobenius Theorem to $A$ restricted to $N_{i} \ominus N_{i-1}$.

Theorem 3.4. Let $\mathcal{S}$ be an indecomposable semigroup in $M_{n}\left(\mathbb{R}^{+}\right)$with $\rho(S)=1$ for all $S$ in $\mathcal{S}$. If a minimal idempotent $E$ in $\mathcal{S}$ satisfies the condition that for all $S$ in $\mathcal{S}$

$$
S E=E S
$$

then there exists a positive diagonal similarity D, such that

$$
\left\|D^{-1} S D\right\| \leq 1 \text { for all } S \in \mathcal{S} \text {. }
$$

Proof. Let $E$ be a minimal idempotent satisfying the conditions of the Theorem. Then minimality implies that there are standard subspaces $\left\{M_{i}\right\}_{i=1}^{r}$ such that $\mathbb{R}^{n}=$ $M_{1} \oplus M_{2} \oplus \cdots \oplus M_{r}$ and that, with respect to this decomposition, $E$ is the direct sum of indecomposable rank-one matrices. So

$$
E=\left[\begin{array}{llll}
x_{1} y_{1}^{*} & & & \\
& x_{2} y_{2}^{*} & & \\
& & \ddots & \\
& & & x_{r} y_{r}^{*}
\end{array}\right]
$$

where each $x_{i}$ and $y_{i}$ is a vector with strictly positive entries and $y_{i}^{*} x_{i}=1$ for all $i=1,2, \ldots, r$ (see [2]). Let $D$ be the positive diagonal matrix such that (restricted to $\left.M_{i}\right) D^{2} x_{i}=y_{i}$. Then

$$
P=D E D^{-1}=\left[\begin{array}{llll}
z_{1} z_{1}^{*} & & & \\
& z_{2} z_{2}^{*} & & \\
& & \ddots & \\
& & & z_{r} z_{r}^{*}
\end{array}\right]
$$

where each $z_{i}$ is a unit vector with strictly positive entries. By applying this diagonal similarity to our semigroup, we may now assume that $E=P$ is self-adjoint and we need to show that all elements of $\mathcal{S}$ are contractions. 
Denote the block matrix of $S$ in $\mathcal{S}$ with respect to the decomposition $\mathbb{R}^{n}=$ $M_{1} \oplus M_{2} \oplus \cdots \oplus M_{r}$ by $\left[S_{i, j}\right]$. Now $S P=P S$, so:

$$
\begin{aligned}
{\left[\begin{array}{cccc}
S_{1,1} & S_{1,2} & & S_{1, r} \\
S_{2,1} & S_{2,2} & & S_{2, r} \\
& & \ddots & \\
S_{r, 1} & S_{r, 2} & & S_{r, r}
\end{array}\right]\left[\begin{array}{llll}
z_{1} z_{1}^{*} & & & \\
& z_{2} z_{2}^{*} & & \\
& & \ddots & \\
& & & z_{r} z_{r}^{*}
\end{array}\right] } \\
=\left[\begin{array}{lllll}
z_{1} z_{1}^{*} & & & \\
& z_{2} z_{2}^{*} & & \\
& & \ddots & \\
& & & z_{r} z_{r}^{*}
\end{array}\right]\left[\begin{array}{llll}
S_{1,1} & S_{1,2} & & S_{1, r} \\
S_{2,1} & S_{2,2} & & S_{2, r} \\
& & \ddots & \\
& & & \\
S_{r, 1} & S_{r, 2} & & S_{r, r}
\end{array}\right] .
\end{aligned}
$$

Multiplying, we obtain that

$$
S_{i, j} z_{j} z_{j}^{*}=z_{i} z_{i}^{*} S_{i, j}
$$

For these rank-one matrices to be equal, it must be the case that there exist constants $\alpha_{i, j}>0$, for $i, j=1,2, \ldots r$ such that $S_{i, j} z_{j}=\alpha_{i, j} z_{j}$.

Let $\widehat{z_{i}}$ denote the vector in $\mathbb{R}^{n}$ whose $i$-th entry with respect to the decomposition $\mathbb{R}^{n}=M_{1} \oplus M_{2} \oplus \cdots \oplus M_{r}$ is $z_{i}$ and whose other entries are zero. Next, consider the subspace $P\left(\mathbb{R}^{n}\right)$, which is the span of $\left\{\widehat{z}_{i}\right\}_{i=1}^{r}$. Since, for each $S$ in $\mathcal{S}, S P=P S$, this subspace is reducing for $\mathcal{S}$. With respect to the decomposition $\mathbb{R}^{n}=P\left(\mathbb{R}^{n}\right) \oplus$ $P^{\perp}\left(\mathbb{R}^{n}\right)$, each $S$ in $\mathcal{S}$ has the form

$$
S=\left[\begin{array}{cc}
A_{S} & 0 \\
0 & B_{S}
\end{array}\right]
$$

where $A_{S}=\left[\alpha_{i, j}\right]$ is a matrix with non-negative entries and $B_{S}$ is some (possibly not positive) matrix.

The minimality of the idempotent $E$ implies that each $A_{S}$ is invertible, and that $\left\{\left.P S P\right|_{P \mathbb{R}^{n}}: S \in \mathcal{S}\right\}$ is a compact semigroup of invertible positive matrices and hence a group. By Lemma 5.1.11 of [9] this group must consist of monomial matrices. However, this group is also self-adjoint, which implies that the group consists of permutation matrices

This means that exactly one $\left[\alpha_{i, j}\right]$ in any row or column is nonzero. However, since $S_{i, j} z_{j}=\alpha_{i, j} z_{j}$ and $z_{j}$ is strictly positive, if $\alpha_{i, j}=0$, then $S_{i, j}=0$. Thus, with respect to the decomposition $\mathbb{R}^{n}=M_{1} \oplus M_{2} \oplus \cdots \oplus M_{r}, S=\left[S_{i, j}\right]$ is block monomial (at most one block in any row or column of the block matrix with respect to this decomposition is non-zero).

Each entry of each $A_{S}$ is either 0 by 1 . This implies that

$$
\left(S_{i, j}^{*} S_{i, j}\right) z_{j}=z_{j}
$$

By Lemma 3.3, a positive matrix admitting a positive eigenvector whose corresponding eigenvalue is 1 must have spectral radius 1 , and so

$$
\left\|S_{i, j}\right\|^{2}=\left\|S_{i, j}^{*} S_{i, j}\right\|=\rho\left(S_{i, j}^{*} S_{i, j}\right)=1
$$

This implies that $S$ has norm 1 .

Corollary 3.5. Let $\mathcal{S}$ in $M_{n}\left(\mathbb{R}^{+}\right)$be a semigroup generated by an indecomposable postitive matrix $A$ with $\rho(A)=1$. Then there exists a positive diagonal similarity 
$D$ such that

$$
\left\|D^{-1} S D\right\| \leq 1 \text { for all } S \in \mathcal{S} \text {. }
$$

Proof. The Perron-Frobenius Theorem (see Corollary 5.2.13 of [9]) guarantees the existence of a minimal idempotent $E$ satisfying the conditions of Theorem 3.4.

The hypothesis that our semigroup is indecomposable (in Theorem 3.4 and Corollary 3.5) can be removed, if we are willing to replace the bound of 1 by $1+\varepsilon$ for $\epsilon>0$. In general, we have the following:

Theorem 3.6. Let $\mathcal{S}$ be a compact semigroup in $M_{n}\left(\mathbb{R}^{+}\right)$, and let

$$
\left\{M_{0}=\{0\} \subset M_{1} \subset M_{2} \subset \cdots \subset M_{k}=\mathbb{R}^{n}\right\}
$$

be a chain of standard invariant subspaces $\mathcal{S}$. Let $P_{N_{i}}$ denote the projection onto $N_{i}=M_{i} \ominus M_{i-1}$ and let $\mathcal{S}_{i}=\left.P_{N_{i}} \mathcal{S}\right|_{N_{i}}$. If there exists $\gamma \in \mathbb{R}^{+}$and positive diagonal invertible matrices $D_{i}$ acting on $N_{i}$ such that

$$
\left\|D_{i}^{-1} S_{i i} D_{i}\right\| \leq \gamma \text { for all } S_{i i} \text { in } \mathcal{S}_{i}
$$

then for all $\varepsilon>0$ there exists a positive diagonal invertible matrix $D$ such that

$$
\left\|D^{-1} S D\right\| \leq \gamma+\varepsilon \text { for all } S \text { in } \mathcal{S} .
$$

Proof. Given $\varepsilon>0$, by compactness there is a $\delta>0$ such that the matrix $D$, which is block diagonal with respect to the decomposition $\mathbb{R}^{n}=N_{1} \oplus N_{2} \oplus \cdots N_{k}$ defined as follows

$$
D=D_{1} \oplus \delta D_{2} \oplus \delta^{2} D_{3} \oplus \cdots \oplus \delta^{k-1} D_{k}
$$

has the required property.

Corollary 3.7. Let $\mathcal{S}$ in $M_{n}\left(\mathbb{R}^{+}\right)$be a semigroup generated by a positive matrix $A$ with $\rho(A)=1$ and let $\varepsilon>0$. Then there exists a positive diagonal similarity $D$ such that

$$
\left\|D^{-1} S D\right\| \leq 1+\varepsilon \text { for all } S \in \mathcal{S} .
$$

In closing, we note that, while we have looked only at the finite-dimensional case, Theorem 3.4 admits an infinite-dimensional analogue whose proof is almost identical to the one given above.

Theorem 3.8. Let $\mathcal{S}$ be an indecomposable semigroup acting on $L^{2}(X, \mu)$, where $X$ is Hausdorff-Lindelöf and $\mu$ is a $\sigma$-finite regular Borel measure on $X$. If $\mathcal{S}$ consists of positive compact operators with $\rho(S)=1$ for all $S$ in $\mathcal{S}$ and a minimal idempotent $E$ in $\mathcal{S}$ satisfies the condition that for all $S$ in $\mathcal{S}$

$$
S E=E S
$$

then there exists a positive invertible multiplication operator $M_{\varphi}\left(\right.$ so $\left.\varphi \in L^{\infty}(X, \mu)\right)$, such that

$$
\left\|M_{\varphi}^{-1} S M_{\varphi}\right\| \leq 1 \text { for all } S \in \mathcal{S} \text {. }
$$

The proof follows as the proof of Theorem 3.4. The continuity of spectral radius on compact operators [7] is needed, and finite-dimensional results on the structure of positive idempotents and the Perron-Frobenius Theorem are replaced by infinitedimensional versions $([10],[5])$. 


\section{REFERENCES}

[1] H. Auerbach, Sur les groupes bornés de substitutions linéares, C. R. Acad. Sci. Paris, 195 (1932), 1367-1369.

[2] A. Berman and R. Plemmons, Nonnegative Matrices in Mathematical Sciences, Academic Press, New York, 1979

[3] G. Frobenius, Uber Matrizen aus nicht negativen Elementen, Sitzungsberichte Akademie der Wissenschaften zu Berlin (1912), 456-477.

[4] F. John, Extremum problems with inequalities as subsidiary conditions, Studies and Essays Presented to R. Courant on his 60th Birthday, January 8, (1948), 187-204. Interscience Publishers, Inc., New York, N. Y., 1948.

[5] M. Krein and M. Rutman, Linear Operators leaving invariant a cone in a Banach space, Uspehi Matem. Nauk (N.S.) 3 (1948), 3-95 (Russian); Amer. Math. Soc. Translation 26, 1950 (English).

[6] L. Livshits, G. MacDonald and H. Radjavi, Universal Bounds for Matrix Semigroups, Studia Mathematica, 203 (2011), 69-77.

[7] J. Newburgh, The variation of spectra, Duke Math. J. 18 (1951), 165-176.

[8] O. Perron, Zur Theorie der Matrizen, Math. Ann. 64 (1907), 248-263.

[9] H. Radjavi and P. Rosenthal, Simultaneous Triangularization, Universitext, Springer, (2000).

[10] Y. Zhong, Functional positivity and invariant subspaces of operators, Houston J. Math. 19 (1993), 239-262.

Leo Livshits: Department of Mathematics, Colby College, Waterville, Maine 04901, USA

E-mail address: llivshi@colby.edu

Gordon MacDonald: Department of Math and Stats, University of Prince Edward Island, Charlottetown, PE, C1A 4P3, Canada

E-mail address: gmacdonald@upei.ca

Laurent Marcoux: Department of Pure Mathematics, University of Waterloo, on CANADA, N2L 3G1

E-mail address: lwmarcoux@uwaterloo.ca

Heydar Radjavi: Department of Pure Mathematics, University of Waterloo, on CANADA, N2L 3G1

E-mail address: hradjavi@uwaterloo.ca 